Winter Meeting - Joint meeting between the Nutrition Society and the Royal Society of Medicine, 11-12 December 2012,

\title{
Statin prescription and dietary imprudence
}

\author{
S. F. McAleer ${ }^{1}$, C. E. Neville ${ }^{2}$, M. C. McKinley ${ }^{2,3}$, J. V. V. Woodside $^{2,3}$, M. A. Tully ${ }^{1,3,2}$ \\ and M. E. Cupples ${ }^{1,2,3}$ \\ ${ }^{1}$ Dept of General Practice and Primary Care, Queen's University Belfast, Belfast, UK, BT9 7HR, ${ }^{2}$ Centre for Public Health, \\ Queen's University Belfast, Belfast, UK, BT12 6BJ and ${ }^{3}$ UKCRC Centre of Excellence for Public Health (NI), Belfast, \\ BT12 6BJ, UK
}

In efforts to obtain optimal control of serum cholesterol, statin prescriptions have increased exponentially over the past 10 years in the $\mathrm{UK}^{(1)}$. NICE guidelines recommend that statin prescription should be accompanied by the delivery of advice to follow a healthy diet, but little is known about reported lifestyle behaviour changes after statin prescription. This study examined reported changes in diet following initiation of statin therapy in a UK primary care population.

Twelve general practices were selected in greater Belfast and all agreed to participate. Over a four month period, eligible patients were selected using a specified search strategy, these being those who were fully registered in the practices, aged $>18 \mathrm{y}$, given a prescription for a statin medication and with no history of statin use. Following informed consent, each participant was posted a 4-day food diary and, 72-96 hours later, were contacted by telephone to confirm their eligibility, ask questions about their smoking habit, lifestyle advice received, recent changes in diet and past medical history ${ }^{(2)}$. This initial contact was timed to take place four weeks after their first prescription was issued (Month 1). Three months later a second food diary was posted and the telephone questionnaire was repeated (Month 4). Food diaries were analysed using the Weighed Intake Software Programme (WISP ${ }^{\circledR}$ version 3.0, Tinuviel Software, Warrington, UK). Ethical approval was obtained from the School of Medicine and Dentistry Research Ethics Committee, Queen's University Belfast. Paired t-tests were used to examine changes in dietary intake between Month 1 and Month 4.

Following a single mailing, 122 of 384 (32\%) potentially eligible participants responded, with $n 109$ participants completing both telephone questionnaires and $n 92$ participants completing both food diaries. No significant changes in dietary intake were evident between the two time points, although there was a trend towards a reduction in saturated fat $(P=0.09)$ and an increase in polyunsaturated fat $(P=0.06)$.

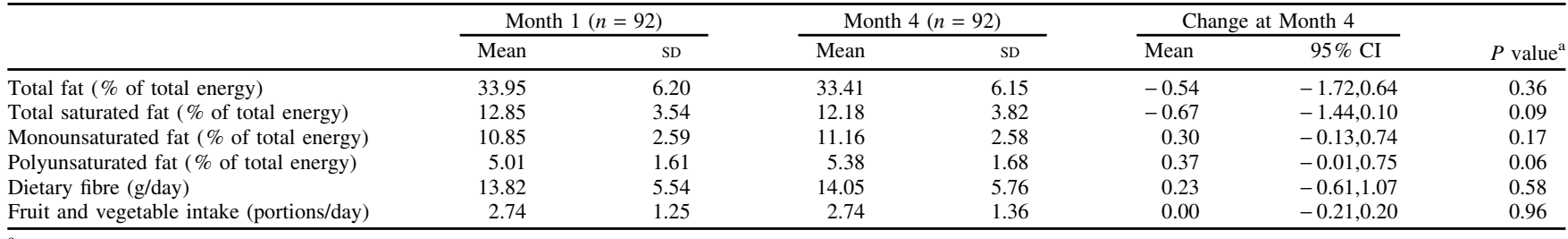

${ }^{\mathrm{a} P}$ value for difference between month 1 and month 4 using paired samples t-test.

From this study, statin prescription in the UK is not associated with significant dietary improvement, suggesting that an increased emphasis on the importance of diet is needed in consultations between healthcare professionals and patients.

1. Tonelli M, Lloyd A, Clement F et al. (2011) CMAJ 183, 1189-1202.

2. Roe L, Strong C, Whiteside C et al. (1994) Fam Pract 11, 375-381. 